\title{
Automated Measurements of Retinal Bifurcations
}

\author{
B. Al-Diri ${ }^{1}$ and A. Hunter ${ }^{1}$ \\ ${ }^{1}$ University of Lincoln, Computing and Informatics, Lincoln, UK
}

\begin{abstract}
This paper presents an analysis of the bifurcations of retinal vessels. The angles and relative diameters of blood vessels in 230 bifurcations were measured using a new automated procedure, and used to calculate the values of several features with known theoretical properties. The measurements are compared with predictions from theoretical models, and with manual measurements. The automated measurements agree with the theoretical prediction measurements with slightly different bias. The automated method can measure a large number of retinal bifurcations very rapidly, and may be useful in correlating bifurcation geometry with clinical conditions.
\end{abstract}

Keywords - Retinal vessel, Bifurcation, Junction, vessel width

\section{INTRODUCTION}

The properties of the vascular network, including the pattern of branching, have been studied for many decades, and a significant body of both theory and experiment has been produced. The vascular network, however, is determined by a complex set of physiological demands, making it difficult to understand, and no fully comprehensive theory has yet emerged. A detailed analysis of the arterial branching prosperity was not achieved until [1]. Nearly half a century passed until excellent studies dealt with measurement of the vascular branching features, and the estimation of branching patterns $[2,3]$. These theoretical studies, while founded on sound physiological principles, have not been extensively compared with experimental data, due to the inherent difficulty in extracting accurate quantitative measurements of bifurcation features [4].

In the retina, a large number of bifurcations can be studied in vivo, and the problem is somewhat simplified as the vessels lie largely in the retinal plane, allowing two dimensional measurements to be taken [5]. However, measuring the bifurcation features accurately remains extremely difficult, whether in vivo or in vitro [4].

In this paper we present an algorithm that can automatically measure a very large number of bifurcations in a very short time, compared to manual methods. We compare the results with theoretical predictions and manual measurements, showing good agreement.

\section{Materials And Methods}

\section{A. Theoretical analysis of the retinal vasculature}

Several authors have proposed that the arterial branching pattern is governed by optimality principles: minimization of the lumen surface, lumen volume, pumping power and endothelial drag. The basic bifurcation features are vessel diameters and bifurcation angles; see Fig. 1. The branching angles and relative diameters are proposed to be sodesigned to optimize the efficiency of the entire vascular network - bearing in mind the fundamental requirement to service the requirements of the organ. More details can be found in $[3,6]$.

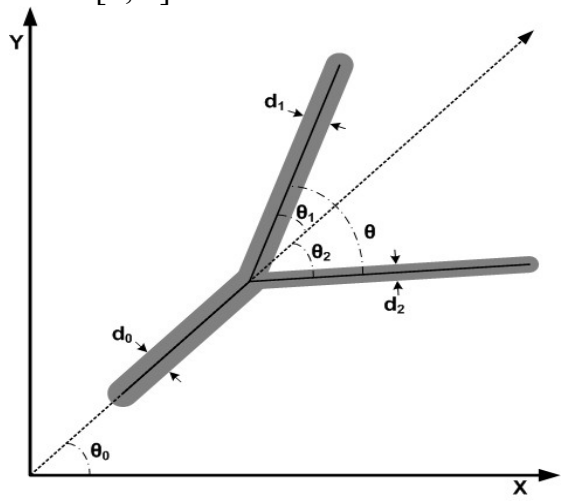

Fig. 1 The basic bifurcation features: diameters $\left(\mathrm{d}_{\mathrm{i}}\right)$ and bifurcation angles $\left(\theta, \theta_{\mathrm{i}}\right)$.

Several of these principles predict that the branching geometry can be predicted based upon the $k$ power law [7], where $k$ is also known as the junction exponent:

$r_{0}^{k}=r_{1}^{k}+r_{2}^{k}$,

where $r$ is the radius of the vessel, and $\mathrm{d}=2 r$ is the vessel diameter. The power laws can be re-expressed with respect to the asymmetry ratio [8],

$$
\alpha=\frac{d_{2}^{2}}{d_{1}^{2}},
$$

These features include the area ratio:

$\beta=\frac{\left(d_{1}^{2}+d_{2}^{2}\right)}{d_{0}^{2}}=(1+\alpha)\left(1+\alpha^{k / 2}\right)^{-2 / k}$ 
the bifurcation index:

$\lambda=\frac{d_{2}}{d_{1}}$

the diameter ratios:

$\lambda_{1}=\frac{d_{1}}{d_{0}}=\left(1+\alpha^{k / 2}\right)^{-1 / k}$ and $\lambda_{2}=\frac{d_{2}}{d_{0}}=\alpha^{1 / 2}\left(1+\alpha^{k / 2}\right)^{-1 / k}$

and the bifurcation angles for minimum pumping power and lumen volume:

$\cos \theta_{1}=\frac{\lambda_{1}^{-4}+1-\lambda^{4}}{2 \lambda_{1}^{-2}} ; \cos \theta_{2}=\frac{\lambda_{1}^{-4}+\lambda^{4}-1}{2 \lambda^{2} \lambda_{1}^{-2}}$

and for minimum drag and lumen surface:

$\cos \theta_{1}=\frac{\lambda_{1}^{-2}+1-\lambda^{2}}{2 \lambda_{1}^{-1}} ; \cos \theta_{2}=\frac{\lambda_{1}^{-2}+\lambda^{2}-1}{2 \lambda \lambda_{1}^{-1}}$

\section{B. Manual Measurement of Bifurcation Features}

In [4], 51 normal human retinal arterial bifurcations were measured manually and compared with theoretical predictions [4]. These measurements are used for comparison and discussion in this paper. The images were enlarged and traced on white sheet, with measurements taken from the tracing; the magnification level was approximately X100, but varied between junctions. Diameters were measured with the aid of either vernier calipers (MTI Corp., New York) or a comparator (MTI Corp.) with a magnifying lens. Branching angles were measured by drawing the center lines of the vessels near the junction and then using a protractor to read the angles between them.

\section{Automated Measurement of Bifurcation Features}

An automated algorithm for segmenting and measuring retinal vessels, by growing a "Ribbon of Twins" active contour model, was used [9]. This algorithm uses two pairs of contours to capture each vessel edge, while maintaining width consistency; see fig. 2 . The algorithm is robust, and can accurately locate vessel edges under difficult conditions and yields precise vessel width measurements, with subpixel average width errors [10].

The DRIVE database [11] was used to test the performance of the algorithm. It was obtained from a diabetic retinopathy screening programme in the Netherlands. The screening population subjects ranged from 31 to 86 years of age. The images were captured in digital form from a Canon CR5 non-mydriatic 3CCD camera at $45^{\circ}$ field of view (FOV) of approximately 540 pixels in diameter. The images are in compressed JPEG-format, they are of size $768 \times 584$ pixels, 8 bits per colour channel.

The database consists of 40 images, split into a training and a test set, each containing 20 images; seven contain pathology, namely exudates, haemorrhages and pigment epithelium changes (three in the training set, and four in the test set).

The retinal vessels of the first ten images from the test set were segmented; see Figure 3. The retinal bifurcation features including angles and relative diameters of blood vessels in 230 bifurcations in the retina were measured automatically.

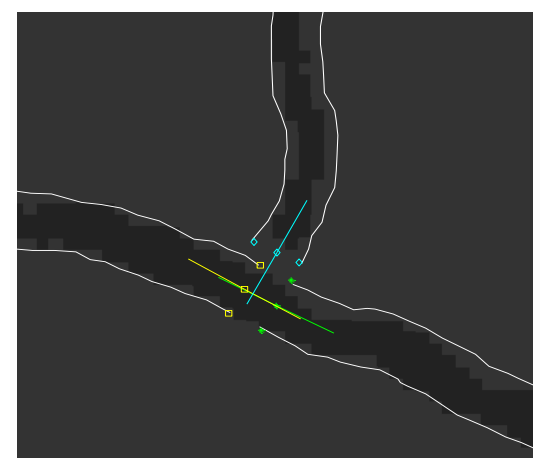

Fig. 2 Vessel segments are automatically segmented and the bifurcation features are calculated. The stars, diamonds and rectangles represent the parent, the first child and the second child respectively.

\section{RESULTS}

The theoretical predictions and the automated bifurcation features are presented in terms of $\beta, \lambda_{1}, \lambda_{2}, \theta_{1}, \theta_{2}$ and $\theta$ against $\alpha$ in Figs. 3-8.

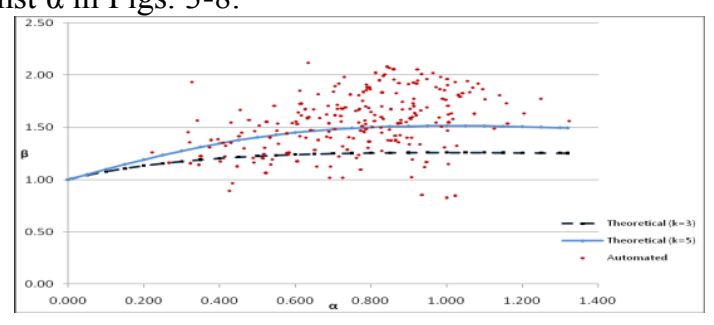

Fig. 3. Measurements of $\beta$ and $\alpha$. The curves are based on the theoretical result in Eq. 3 when $\mathrm{k}$ equals three (dashed line) and five (solid line). The data points are the automated measurements.

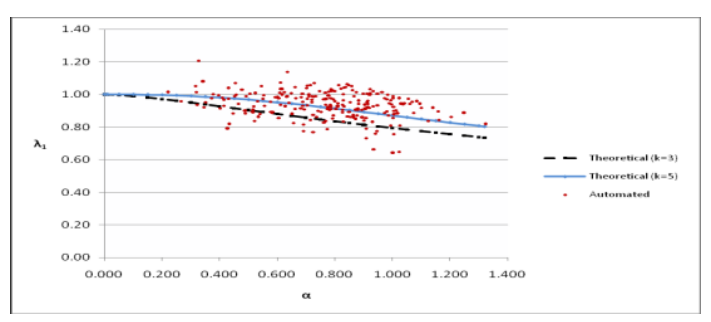

Fig. 4. Measurements of $\lambda_{1}$ and $\alpha$. The curves are based on the theoretical result in Eq. 5 when $\mathrm{k}$ equals three (dashed line) and five (solid line). The data points are the automated measurements. 


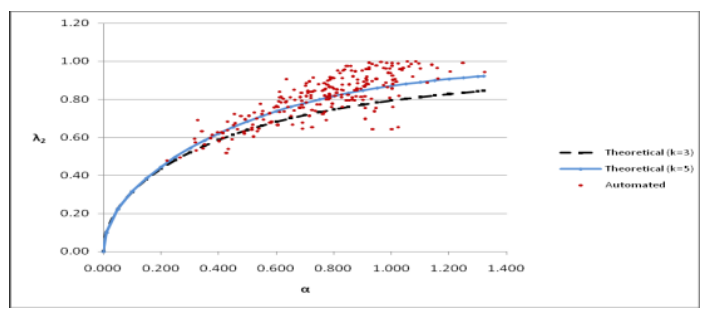

Fig. 5. Measurements of $\lambda_{2}$ and $\alpha$. The curves are based on the theoretical result in Eq. 5 when k equals three (dashed line) and five (solid line). The data points are the automated measurements.

Figs. 6, 7 and 8 can be used to map out measured retinal bifurcations to determine whether or not they fall between the theoretical predicted curves, therefore satisfying all four principles. The curves are based on conditions of minimum lumen surface and drag (solid line) and minimum lumen volume and pumping power (dashed line), which are based on the theoretical result in Eqs. 6 and 7.

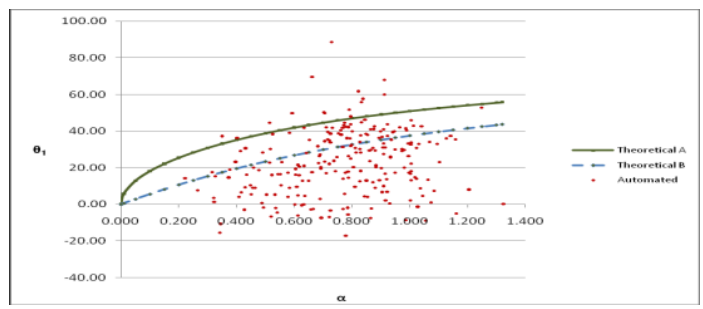

Fig. 6 Measurements of $\theta_{1}$ and $\alpha$. The curves represent the theoretical predictions based on conditions of minimum lumen surface and drag (solid line), and minimum lumen volume and pumping power (dashed line). The data points are the automated measurements.

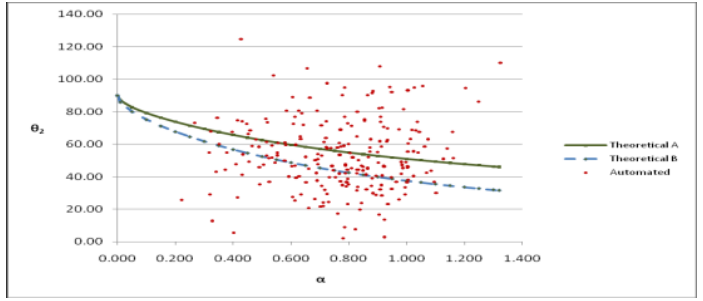

Fig. 7 Measurements of $\theta_{2}$ and $\alpha$. The curves represent the theoretical predictions. The data points are the automated measurements.

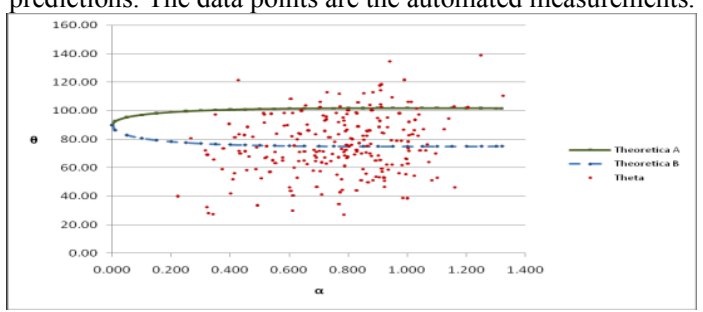

Fig. 8 Measurements of $\theta\left(\theta_{1}+\theta_{2}\right)$ and $\alpha$. The curves represent the theoretical predictions. The data points are the automated measurements.

\section{CONCLUSIONS}

The correlation between experimentally measured features of vascular bifurcations and the theoretical predictions is characterized by a large amount of variability. This variability may be due to normal biological scatter [4], the values of the power law exponent $k$ [6], experimental measurement errors, the scatter in physiological data [12] or the presence of a multifractal pattern which suggests that the bulk of the scatter is because of vascular design variability [13]. The noticed variations could also be attributed to the fact that some of the used images were of patients with variable grades of diabetic retinopathy, thus their geometrical features could deviate more from the theoretical optimum due to pathological alterations of the applied forces on the retinal vessels. Table 1 shows the percentage of the experimental data points within $\pm 10 \%$ deviation around the theoretical curves; the manual measurement performance is taken from [4], the automated measurements were taken from the DRIVE database. There is thus an unknown degree of intra-experimental variation in the results.

Table 1 A strip of $\pm 10 \%$ deviation around theoretical curves covers the given percentage of the manual and automated measurements.

\begin{tabular}{lll}
\hline Features & Manual & Automated \\
\hline$\beta$ & - & $20 \%$ \\
$\lambda_{1}$ & $64 \%$ & $39 \%$ \\
$\lambda_{2}$ & $64 \%$ & $39 \%$ \\
$\theta$ & $60 \%$ & $60 \%$ \\
$\theta_{1}$ & $32 \%$ & $32 \%$ \\
$\theta_{2}$ & $48 \%$ & $43 \%$ \\
\hline
\end{tabular}

A strip of $\pm 20 \%$ deviation covers $55 \%$ of $\beta$ data points from the manual measurements [4] and $38 \%$ of automated measurements. The bifurcation angle measurements of the manual method and the automated algorithm are very similar. In contrast, there is a noticeable difference between the relative diameter measurement features for the automated and manual techniques. The sources of biological scatter include the variable curvature, and non-uniform width of vessels [4]. The automated algorithm calculates the vessel width integrated along a short length of the vessel at the bifurcation [10], and has been shown in evaluation [14] to provide more accurate width measurement than alternative algorithms.

The manual measurement method is subject to operator error, including inter- and intra- observer errors. Although this variation is considered [4] to be the smallest contributor to the measurement variation, it may have a subtle effect on the variation when the observer's perspective is affected by an understanding of the basic theoretical rules (e.g. the value of $\beta$ is greater than one as the parent should be wider 
than the children, the value of $\lambda$ is less than one, the branching should come off one on each side [4]). In reality, in some cases $\beta$ is less than $1, \lambda$ is greater than 1 and/or $\theta_{1}$ may be negative (one or more of these conditions is true in $21 \%$ of cases according to [5]; see Table 2 .

Table 2 Statistical description of the automated measurements.

\begin{tabular}{|c|c|c|c|c|c|c|c|c|c|}
\hline & & $\alpha$ & $\beta$ & $\lambda$ & $\lambda 1$ & $\lambda 2$ & $\mathrm{k}$ & $\theta_{1}$ & $\theta_{2}$ \\
\hline \multirow{4}{*}{ 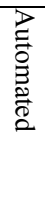 } & Mean & 0.77 & 1.56 & 0.87 & 0.94 & 0.81 & 4.95 & 23 & 53 \\
\hline & Max & 1.32 & 2.12 & 1.15 & 1.21 & 1.00 & 28.95 & 89 & 125 \\
\hline & Min & 0.22 & 0.83 & 0.47 & 0.64 & 0.48 & 0 & -17 & 2 \\
\hline & Stdev & 0.21 & 0.27 & 0.13 & 0.08 & 0.12 & 5.11 & 18 & 22 \\
\hline
\end{tabular}

In contrast, the automated measurements are not subject to unconscious "confirmation bias" of an operator. This may help to explain the lower numbers of measurements close to the theoretical predicted values for $\beta, \lambda_{1}$ and $\lambda_{2}$ features in Table 1. The standard theoretical predictions are based upon a value $k=3$, which corresponds to certain optimality principles. However, experimental analysis suggests that in reality $k$ is usually in the range 1-5 [7]. We have compared the automated measurements with the theoretical predictions when $k$ equals 4 and 5; see Table 3 . The percentage of the automated measurements in range increases with $k$, supporting the contention that the real $k$ exponent value in the vessel network is greater than 3 .

Table 3. The theoretical predictions calculated using different power values (k) 3, 4 and 5. Strips of $\pm 10 \%$ deviation around theoretical curves cover the given percentage of the automated measurements.

\begin{tabular}{llll}
\hline Features & $\begin{array}{l}\text { Automated } \\
\text { (when } \mathrm{k}=3)\end{array}$ & $\begin{array}{l}\text { Automated } \\
\text { (when } \mathrm{k}=4)\end{array}$ & $\begin{array}{l}\text { Automated } \\
\text { (when } \mathrm{k}=5)\end{array}$ \\
\hline$\beta(20 \%)$ & $38 \%$ & $60 \%$ & $71 \%$ \\
$\beta$ & $20 \%$ & $32 \%$ & $40 \%$ \\
$\lambda_{1}$ & $39 \%$ & $64 \%$ & $72 \%$ \\
$\lambda_{2}$ & $39 \%$ & $64 \%$ & $74 \%$ \\
\hline
\end{tabular}

The branching angles have a higher degree of scatter because the vessels are not always straight in the bifurcation region [6]. Neither the manual measurements nor the automated measurements are fully consistent with the theoretical predictions. This disagreement may occur due to measurement error [4], differential influence of the principles upon the actual values, or noise; see figs. 6, 7 and 8.

To provide a more meaningful comparison between theoretical predictions and experimental results, a larger set of experimental data is required. This would support a more in-depth analysis of the sources of variation of experimental measurements from existing models, perhaps leading to a more sophisticated understanding of the principles underlying the vascular network geometry. This in turn may allow the construction of more effective algorithms to resolve junction configurations, such as that presented in [15].

\section{REFERENCES}

1. Murray C. (1926). "The physiological principle of minimum work applied to the angle of branching arteries". J Gen Physiol. 9:835-841

2. Rodbard, S. (1975). "Vascular calibre". Cardiology, 60, 4-49.

3. Zamir M. (1976). "Optimality principles in arterial branching". J Theor Biol. 62:227-251

4. Zamir M, Medeiros J, Cunningham TK. (1979). "Arterial bifurcations in the human retina," J Gen Physiol. 74:537-548

5. Al-Diri, B. (2008). "Analysis of Blood Vessels for Characterization of Diabetic Retinopathy”. PhD in Computer Vision, Department of Computing and Informatics, University of Lincoln, UK.

6. Zamir M, Medeiros J. (1982). "Arterial branching in man and monkey". J Gen Physiol. 79:353-360.

7. Zamir, M., Sinclair, P. \& Wannacott, T.H. (1992). "Relation between diameter and flow in major branches of the arch of the aorta". Journal Biomechanics, 25, 1303-1310.

8. Zamir, M. (1978). "Nonsymmetrical bifurcations in arterial branching". J Gen Physiol. 72, 837-845.

9. Al-Diri, B., Hunter, A. (2005). "A ribbon of twins for extracting vessel boundaries," IFMBE Proc. 3rd European Medical and Biological Engineering Conference EMBEC'05, November, 2025, Prague, Czech Republic.

10. Al-Diri, B., Hunter, A., Steel, D. (2009). "An Active Contour Model for Segmenting and Measuring Retinal Vessels", IEEE In Press.

11. Staal, J., Abrmoff, M.D., Niemeijer, M., Viergever, M.A. \& Ginneken, B.V., (2004). "Ridge based vessel segmentation in color images of the retina", IEEE Transactions on Medical Imaging, 23, 501-509.

12. Zamir, M. (2001a). Arterial branching within the confines of fractal L-system formalism. J Gen Physiol. 118, 267-275.

13. Zamir, M. (2001b). "Fractal dimensions and multifractility in vascular branching". Journal of Theoretical Biology, 212, 183190.

14. Al-Diri, B., Hunter, A., Steel, D., \& Habib, M., Hudaib, T., \& Berry, S. (2008). "Review - a reference data set for retinal vessel profiles," in Proc. $30^{\text {th }}$ Annual International Conference of the IEEE Engineering in Medicine and Biology Society, August 20-24, British Columbia, Canada.

15. Al-Diri, B., Hunter, A., Steel, D., \& Habib, M. (2008). "Joining retinal vessel segments," Proc. 8th IEEE International Conference on BioInformatics and BioEngineering (BIBE), October 8-10, Athens, Greece.

Author: Bashir Al-Diri

Institute: The University of Lincoln

Street: Brayford

City: Lincoln

Country: UK

Email: baldiri@lincoln.ac.uk 